\title{
Geometric aspects of certain second order differential systems in particle physics
}

\author{
Vladimir Balan ${ }^{1, *}$, Viktor Red'kov ${ }^{2, * *}$, Elena Ovsiyuk ${ }^{3, * * *}$, and Nina Krylova ${ }^{4, * * * *}$ \\ ${ }^{1}$ University Politehnica of Bucharest, Romania \\ 313 Splaiul Independentei, Bucharest, Romania \\ ${ }^{2}$ Institute of Physics, National Academy of Science of Belarus \\ ${ }^{3}$ Mozyr State Pedagogical University, Belarus \\ ${ }^{4}$ Belarusian State University, Minsk, Belarus
}

Abstract. We consider the problem of the spin 1 particle with anomalous magnetic moment in an external Coulomb field, in non-relativistic approximation. The structural stability of the extended second order ODE system is studied.

\section{Introduction}

It is known that, in the framework of the theory of relativistic wave equations, one can consider the so-called non-minimal equations, which describe particles with additional electromagnetic characteristics. In particular, the equations for spin $S=1 / 2$ and $S=1$ particles with both electric charge and anomalous magnetic moment were extensively studied [8-10, 12, 16-22, 28]. In the present talk we focus on the spin $S=1$ vector particle with anomalous magnetic moment in the external Coulomb field.

In the case of external Coulomb field, the equation for the vector particle is too complicated, even for the case of an ordinary particle without anomalous moment. This problem has not been solved completely yet.

However, in the non-relativistic limit, the equation for an ordinary vector particle in the Coulomb field can be exactly solved. For this reason, in the present paper we investigate the non-relativistic problem for the particle with anomalous magnetic moment.

The study of the the quantum mechanical problem of a vector particle with anomalous magnetic moment in the external Coulomb field is highly nontrivial, and the description of finding and describing the behavior of its multiple classes of solutions is a challenging concern. To this aim, the relativistic Duffin-Kemmer-Petiau approach is shown to provide a system of 10 radial equations which can be split into two systems of 4 and 6 equations for states with parities $P=(-1)^{j+1}$ and $P=(-1)^{j}$, respectively. The interaction terms which are due to anomalous magnetic moment are present only in the system corresponding to states with parity $P=(-1)^{j}$.

The non-relativistic approximation states with minimal value $j=0$ are described by a second order equation of double confluent Heun type. By imposing the known transcendency

\footnotetext{
*e-mail: vladimir.balan@upb.ro

**e-mail: v.redkov@ifanbel.bas-net.by

***e-mail: e.ovsiyuk@mail.ru

****e-mail: nina-kr@tut.by
} 
condition, we derive the energy spectrum of the structure, $E=-\mathrm{const} / n^{2}$. The numerical values for energies seem to be physically reasonable, though they do not depend on the anomalous magnetic moment.

For states with $j=1,2, \ldots$, we obtain a system of two second order linked differential equations for radial functions. Its Frobenius solutions are constructed, and the convergence of the involved power series with 8- and respectively 9-terms recurrence relations, is studied.

We apply the geometrical method based on the use of KCC-invariants to this problem. The first and the second invariants are determined and their implications on the behavior of the solutions is discussed. It is shown that the different branches of the solutions converge near the singular points $\infty,-\Gamma / 2$, and may either converge or diverge near the singular points with $r=0$. This correlates with the expected behavior of solutions for bound states.

In the last part of the work, the extended system of 10 radial equations implied by the original matrix equation is considered from a SODE - oriented KCC perspective. This system leads to two real second-order 4-subsystems, which can are investigated by using the tools of the Jacobi (structural) stability theory by examining its related geometric KCC-invariants. To this aim, we state several results regarding the framework which embeds the second order ODE system of 4 equations obtained from the real dynamical system of 8 equations. We also note that the KCC theory can be applied as well to the canonically associated 2-nd order 8-dimensional real system obtained via the Geometric Dynamics extension process [30], for which alternative information on the behavior of solutions is provided by the associated KCC invariants.

\section{The extension of the complex Duffin-Kemmer-Petiau equation}

The initial nonminimal relativistic equation that describes a spin 1 particle has the following form ([23]):

$$
\left\{i \beta^{c}\left[i\left(e_{(c)}^{\beta} \partial_{\beta}+\frac{1}{2} j^{a b} \gamma_{a b c}(x)\right)-e^{\prime} A_{c}\right]+\lambda \frac{e}{M} F_{\alpha \beta}(x) P j^{\alpha \beta}(x)-M\right\} \Psi=0,
$$

where the free parameter $\lambda$ is dimensionless, $P$ is the projective linear operator $P=$ $\operatorname{diag}\left(\mathrm{O}_{4}, I_{6}\right)$ which selects the tensor component from the 10-components unknown vector function, and we use the following notations:

$$
M=\frac{m c}{\hbar}, \quad e^{\prime}=\frac{e}{c \hbar}, \quad \Gamma=\lambda \frac{4 \alpha}{M}, \quad \alpha=\frac{e^{2}}{\hbar c}=\frac{1}{137} .
$$

In the spherical tetrad [24], the equation (1) takes the form

$$
\left[\beta^{0}\left(i \partial_{t}+\frac{\alpha}{r}\right)+i\left(\beta^{3} \partial_{r}+\frac{1}{r}\left(\beta^{1} j^{31}+\beta^{2} j^{32}\right)\right)+\frac{1}{r} \Sigma_{\theta, \phi}+\frac{\Gamma}{r^{2}} P j^{03}-M\right] \Phi=0,
$$

where the angular operator is determined by the equality:

$$
\Sigma_{\theta, \phi}=i \beta^{1} \partial_{\theta}+\beta^{2} \frac{i \partial_{\phi}+i j^{12} \cos \theta}{\sin \theta}
$$


with

$$
\begin{array}{lll}
\beta^{0}=\left(\begin{array}{cc}
0 & v \\
v^{t} & a
\end{array}\right), & A=\left(\begin{array}{ccc}
O & B & O \\
-B & O & O \\
O & O & O
\end{array}\right), v=(0,0,0,0,0,0,0,0,0), & B=i\left(\begin{array}{ccc}
1 & 0 & 0 \\
0 & 1 & 0 \\
0 & 0 & 1
\end{array}\right), \\
\beta^{1}=\frac{1}{\sqrt{2}}\left(\begin{array}{cc}
0 & v \\
v^{t} & A
\end{array}\right), & A=\left(\begin{array}{ccc}
O & O & B \\
O & O & O \\
-B & O & O
\end{array}\right), v=i(0,0,0,-1,0,1,0,0,0), & B=\left(\begin{array}{ccc}
0 & 1 & 0 \\
1 & 0 & 1 \\
0 & 1 & 0
\end{array}\right), \\
\beta^{2}=\frac{1}{\sqrt{2}}\left(\begin{array}{cc}
0 & v \\
v^{t} & A
\end{array}\right), & A=\left(\begin{array}{ccc}
O & O & B \\
O & O & O \\
-B & O & O
\end{array}\right), v=(0,0,0,1,0,1,0,0,0), & B=i\left(\begin{array}{ccc}
0 & -1 & 0 \\
1 & 0 & -1 \\
0 & 1 & 0
\end{array}\right), \\
\beta^{3}=\left(\begin{array}{cc}
0 & v \\
v^{t} & a
\end{array}\right), & A=\left(\begin{array}{ccc}
O & O & B \\
O & O & O \\
-B & O & O
\end{array}\right), v=i(0,0,0,0,1,0,0,0,0), & B=\left(\begin{array}{ccc}
1 & 0 & 0 \\
0 & 1 & 0 \\
0 & 0 & -1
\end{array}\right) .
\end{array}
$$

Relative to the used basis, the explicit form of the total angular momentum operator components is given by [24]:

$$
j_{1}=l_{1}+\frac{\cos \phi}{\sin \theta} i j^{12}, \quad j_{2}=l_{2}+\frac{\sin \phi}{\sin \theta} i j^{12}, \quad j_{3}=l_{3}, \quad j^{12}=\beta^{1} \beta^{2}-\beta^{2} \beta^{1} .
$$

We shall further use the wave function and the Duffin-Kemmer matrices in cyclic representation [24]. The matrix $i j^{12}$ has the diagonal structure

$$
i j^{12}=\operatorname{diag}\left(0, t_{3}, t_{3}, t_{3}\right), \quad t_{3}=\operatorname{diag}(1,0,-1) .
$$

The form of the projective operator $P$ does not change under transition from Cartesian basis to the cyclic one.

The system of radial equations for an ordinary vector particle in the Coulomb field is well-known [18]. To obtain the generalized system for the vector particle with anomalous magnetic moment, it suffices to specify the additional term in the equation:

$$
\frac{\Gamma}{r^{2}} P j^{03}=\frac{\Gamma}{r^{2}} P\left(\beta^{0} \beta^{3}-\beta^{3} \beta^{0}\right), \quad P j^{03}=\operatorname{diag}\left(M, O_{7}\right), M=\left(\begin{array}{ccc}
0 & 0 & -1 \\
0 & 0 & 0 \\
-1 & 0 & 0
\end{array}\right) .
$$

The structure of the 10-components wave function for the vector particle with quantum numbers $\epsilon, j, m$ is the following

$$
\begin{gathered}
\Psi(x)=\left\{\Phi_{0}(x), \vec{\Phi}(x), \vec{E}(x), \vec{H}(x)\right\}, \\
\Phi_{0}(x)=e^{-i \epsilon t} f_{0}(r) D_{0}, \quad \vec{\Phi}(x)=e^{-i \epsilon t}\left(\begin{array}{c}
f_{1}(r) D_{-1} \\
f_{2}(r) D_{0} \\
f_{3}(r) D_{+1}
\end{array}\right), \\
\vec{E}(x)=e^{-i \epsilon t}\left(\begin{array}{c}
E_{1}(r) D_{-1} \\
E_{2}(r) D_{0} \\
E_{3}(r) D_{+1}
\end{array}\right), \quad \vec{H}(x)=e^{-i \epsilon t}\left(\begin{array}{c}
H_{1}(r) D_{-1} \\
H_{2}(r) D_{0} \\
H_{3}(r) D_{+1}
\end{array}\right),
\end{gathered}
$$

where $D$ stands for the Wigner functions [24]: $D_{\sigma}=D_{-m, \sigma}^{j}(\phi, \theta, 0), \sigma=0,-1,+1$, After performing the needed calculations, one finds the system of radial equations

$$
\begin{aligned}
& \left\{\begin{array}{l}
\left(\frac{d}{d r}+\frac{2}{r}\right) E_{2}-\frac{v}{r}\left(E_{1}+E_{3}\right)-\frac{\Gamma}{r^{2}} f_{2}=m f_{0}, \\
+i\left(\epsilon+\frac{\alpha}{r}\right) E_{1}+i\left(\frac{d}{d r}+\frac{1}{r}\right) H_{1}+i \frac{v}{r} H_{2}=m f_{1}, \\
+i\left(\epsilon+\frac{\alpha}{r}\right) E_{2}-i \frac{v}{r}\left(H_{1}-H_{3}\right)-\frac{\Gamma}{r^{2}} f_{0}=m f_{2}, \\
+i\left(\epsilon+\frac{\alpha}{r}\right) E_{3}-i\left(\frac{d}{d r}+\frac{1}{r}\right) H_{3}-i \frac{v}{r} H_{2}=m f_{3},
\end{array}\right. \\
& \begin{cases}-i\left(\epsilon+\frac{\alpha}{r}\right) f_{1}+\frac{v}{r} f_{0}=m E_{1}, & -i\left(\epsilon+\frac{\alpha}{r}\right) f_{2}-\frac{d}{d r} f_{0}=m E_{2}, \\
-i\left(\epsilon+\frac{\alpha}{r}\right) f_{3}+\frac{v}{r} f_{0}=m E_{3}, & -i\left(\frac{d}{d r}+\frac{1}{r}\right) f_{1}-i \frac{v}{r} f_{2}=m H_{1}, \\
+i \frac{v}{r}\left(f_{1}-f_{3}\right)=m H_{2}, & +i\left(\frac{d}{d r}+\frac{1}{r}\right) f_{3}+i \frac{v}{r} f_{2}=m H_{3} .\end{cases}
\end{aligned}
$$


The spectral equation involving the spatial inversion operator, expressed spherical basis, has two types of solutions:

$$
\begin{array}{ll}
P=(-1)^{j+1}, & f_{0}=0, f_{3}=-f_{1}, f_{2}=0, E_{3}=-E_{1}, E_{2}=0, H_{3}=H_{1} \\
P=(-1)^{j}, & f_{3}=+f_{1}, E_{3}=+E_{1}, H_{3}=-H_{1}, H_{2}=0 .
\end{array}
$$

a) For such solutions, for the states with $P=(-1)^{j+1}$, we obtain from (5) the four equations:

$$
\begin{gathered}
i\left(\epsilon+\frac{\alpha}{r}\right) E_{1}+i\left(\frac{d}{d r}+\frac{1}{r}\right) H_{1}+i \frac{v}{r} H_{2}=m f_{1}, \\
-i\left(\epsilon+\frac{\alpha}{r}\right) f_{1}=m E_{1}, \quad-i\left(\frac{d}{d r}+\frac{1}{r}\right) f_{1}=m H_{1}, \quad 2 i \frac{v}{r} f_{1}=m H_{2} .
\end{gathered}
$$

Here the anomalous magnetic moment does not manifest itself in any way in the external Coulomb field.

The last 4-equations system leads to the following equation for the main function $f_{1}$ :

$$
\left(\frac{d^{2}}{d r^{2}}+\frac{2}{r} \frac{d}{d r}+\left(\epsilon+\frac{\alpha}{r}\right)^{2}-\frac{j(j+1)}{r^{2}}\right) f_{1}=0 .
$$

The same equation arises in the theory of the scalar particle, in the presence of the Coulomb field. Its exact solutions and the corresponding energy spectrum are well-known.

b) For states with parity $P=(-1)^{j}$, we have six equations:

$$
\begin{array}{ll}
-\left(\frac{d}{d r}+\frac{2}{r}\right) E_{2}-2 \frac{v}{r} E_{1}-\frac{\Gamma}{r^{2}} f_{2}=m f_{0}, & i\left(\epsilon+\frac{\alpha}{r}\right) E_{1}+i\left(\frac{d}{d r}+\frac{1}{r}\right) H_{1}=m f_{1}, \\
+i\left(\epsilon+\frac{\alpha}{r}\right) E_{2}-2 i \frac{v}{r} H_{1}-\frac{\Gamma}{r^{2}} f_{0}=m f_{2}, & -i\left(\epsilon+\frac{\alpha}{r}\right) f_{2}-\frac{d}{d r} f_{0}=m E_{2}, \\
-i\left(\epsilon+\frac{\alpha}{r}\right) f_{1}+\frac{v}{r} f_{0}=m E_{1}, & i\left(\frac{d}{d r}+\frac{1}{r}\right) f_{1}+i \frac{v}{r} f_{2}=-m H_{1} .
\end{array}
$$

I. The non-relativistic equations for $j=0$. For states with minimal level $j=0$, the obtained equations have a complicated set of singular points. Therefore a much simpler non-relativistic analogue was derived by excluding from the system

$$
\begin{aligned}
& \frac{1}{m}\left(-\left(\frac{d}{d r}+\frac{2}{r}\right) E_{2}-\frac{\Gamma}{r^{2}} f_{2}\right)=f_{0}, \\
& +i\left(\epsilon+\frac{\alpha}{r}\right) E_{2}-\frac{\Gamma}{r^{2}} f_{0}=m f_{2}, \\
& -i\left(\epsilon+\frac{\alpha}{r}\right) f_{2}-\frac{d}{d r} f_{0}=m E_{2},
\end{aligned}
$$

the non-dynamical variable $f_{0}(r)$ :

$$
\left\{\begin{array}{l}
+i\left(\epsilon+\frac{\alpha}{r}\right) E_{2}-\frac{\Gamma}{m r^{2}}\left(-\left(\frac{d}{d r}+\frac{2}{r}\right) E_{2}-\frac{\Gamma}{r^{2}} f_{2}\right)=m f_{2} \\
-i\left(\epsilon+\frac{\alpha}{r}\right) f_{2}-\frac{1}{m} \frac{d}{d r}\left(-\left(\frac{d}{d r}+\frac{2}{r}\right) E_{2}-\frac{\Gamma}{r^{2}} f_{2}\right)=m E_{2}
\end{array}\right.
$$

and then by introducing the the big and the small components:

$$
f_{2}=\left(B_{2}+M_{2}\right), \quad i E_{2}=\left(B_{2}-M_{2}\right),
$$

separating the rest energy by means of the formal substitution $\epsilon \rightsquigarrow m+E$, where $E$ is the non-relativistic energy. This leads to

$$
\left\{\begin{array}{l}
\left(E+\frac{\alpha}{r}\right)\left(B_{2}-M_{2}\right)-\frac{\Gamma}{m r^{2}}\left[i\left(\frac{d}{d r}+\frac{2}{r}\right)\left(B_{2}-M_{2}\right)-\frac{\Gamma}{r^{2}}\left(B_{2}+M_{2}\right)\right]=2 m M_{2}, \\
\left(E+\frac{\alpha}{r}\right)\left(B_{2}+M_{2}\right)-\frac{1}{m} \frac{d}{d r}\left[-\left(\frac{d}{d r}+\frac{2}{r}\right)\left(B_{2}-M_{2}\right)-\frac{\Gamma}{r^{2}}\left(B_{2}+M_{2}\right)\right]=-2 m M_{2} .
\end{array}\right.
$$


which summed and with neglecting the small component $M_{2}$, provide:

$$
2\left(E+\frac{\alpha}{r}\right) B_{2}-\frac{\Gamma}{m r^{2}}\left(i\left(\frac{d}{d r}+\frac{2}{r}\right)-\frac{\Gamma}{r^{2}}\right) B_{2}+\frac{1}{m} \frac{d}{d r}\left(\frac{d}{d r}+\frac{2}{r}+\frac{i \Gamma}{r^{2}}\right) B_{2}=0 .
$$

Taking into account that from the physical point of view the parameter $\Gamma$ is imaginary, and making the substitution $i \Gamma \rightsquigarrow \Gamma$, we find (while changing the notation as $B_{2}(r) \rightsquigarrow R(r)$ ), the final equation (the double confluent Heun type) reads:

$$
\frac{d^{2} R}{d r^{2}}+\frac{2}{r} \frac{d R}{d r}+\left(2 m\left(E+\frac{\alpha}{r}\right)-\frac{2}{r^{2}}-\frac{4 \Gamma}{r^{3}}-\frac{\Gamma^{2}}{r^{4}}\right) R=0
$$

The study of its singular points $r=0$ and $r=\infty$ and the local Frobenius solutions in the vicinity of the point $r=0$ were constructed in [13].

II. The non-relativistic equations for $j=1,2,3, \ldots$. Emerging from the relativistic equations:

$$
\begin{array}{ll}
-\left(\frac{d}{d r}+\frac{2}{r}\right) E_{2}-2 \frac{v}{r} E_{1}-\frac{\Gamma}{r^{2} f_{2}}=m f_{0}, & i\left(\epsilon+\frac{\alpha}{r}\right) E_{1}+i\left(\frac{d}{d r}+\frac{1}{r}\right) H_{1}=m f_{1}, \\
+i\left(\epsilon+\frac{\alpha}{r}\right) E_{2}-2 i \frac{v}{r} H_{1}-\frac{\Gamma}{r^{2}} f_{0}=m f_{2}, & -i\left(\epsilon+\frac{\alpha}{r}\right) f_{2}-\frac{d}{d r} f_{0}=m E_{2}, \\
-i\left(\epsilon+\frac{\alpha}{r}\right) f_{1}+\frac{v}{r} f_{0}=m E_{1}, & +i\left(\frac{d}{d r}+\frac{1}{r}\right) f_{1}+i \frac{v}{r} f_{2}=-m H_{1},
\end{array}
$$

by excluding the non-dynamical variables $f_{0}$ and $H_{1}$, we get four equations

$$
\begin{aligned}
& +i\left(\epsilon+\frac{\alpha}{r}\right) E_{1}-i\left(\frac{d}{d r}+\frac{1}{r}\right) \frac{1}{m}\left[i\left(\frac{d}{d r}+\frac{1}{r}\right) f_{1}+i \frac{v}{r} f_{2}\right]=m f_{1}, \\
& -i\left(\epsilon+\frac{\alpha}{r}\right) f_{1}-\frac{v}{r} \frac{1}{m}\left[\left(\frac{d}{d r}+\frac{2}{r}\right) E_{2}+2 \frac{v}{r} E_{1}+\frac{\Gamma}{r^{2}} f_{2}\right]=m E_{1}, \\
& +i\left(\epsilon+\frac{\alpha}{r}\right) E_{2}+2 i \frac{v}{r} \frac{1}{m}\left[i\left(\frac{d}{d r}+\frac{1}{r}\right) f_{1}+i \frac{v}{r} f_{2}\right]+\frac{\Gamma}{r^{2}} \frac{1}{m}\left[\left(\frac{d}{d r}+\frac{2}{r}\right) E_{2}+2 \frac{v}{r} E_{1}+\frac{\Gamma}{r^{2}} f_{2}\right]=m f_{2}, \\
& -i\left(\epsilon+\frac{\alpha}{r}\right) f_{2}+\frac{d}{d r} \frac{1}{m}\left[\left(\frac{d}{d r}+\frac{2}{r}\right) E_{2}+2 \frac{v}{r} E_{1}+\frac{\Gamma}{r^{2}} f_{2}\right]=m E_{2},
\end{aligned}
$$

where the big and the small components can be determined by the formulas

$$
f_{1}=\left(\Psi_{1}+\psi_{1}\right), i E_{1}=\left(\Psi_{1}-\psi_{1}\right), \quad f_{2}=\left(\Psi_{2}+\psi_{2}\right), i E_{2}=\left(\Psi_{2}-\psi_{2}\right) .
$$

By separating the rest energy by the substitution $\epsilon=(m+E))$, we infer

$$
\begin{aligned}
& \left(E+\frac{\alpha}{r}\right)\left(\Psi_{1}-\psi_{1}\right)+\left(\frac{d}{d r}+\frac{1}{r}\right) \frac{1}{m}\left[\left(\frac{d}{d r}+\frac{1}{r}\right)\left(\Psi_{1}+\psi_{1}\right)+\frac{v}{r}\left(\Psi_{2}+\psi_{2}\right)\right]=2 m \psi_{1}, \\
& \left(E+\frac{\alpha}{r}\right)\left(\Psi_{1}+\psi_{1}\right)-\frac{v}{r} \frac{1}{m}\left[\left(\frac{d}{d r}+\frac{2}{r}\right)\left(\Psi_{2}-\psi_{2}\right)+2 \frac{v}{r}\left(\Psi_{1}-\psi_{1}\right)+\frac{i \Gamma}{r^{2}}\left(\Psi_{2}+\psi_{2}\right)\right]=-2 m \psi_{1}, \\
& \left(E+\frac{\alpha}{r}\right)\left(\Psi_{2}-\psi_{2}\right)-2 \frac{v}{r} \frac{1}{m}\left[\left(\frac{d}{d r}+\frac{1}{r}\right)\left(\Psi_{1}+\psi_{1}\right)+\frac{v}{r}\left(\Psi_{2}+\psi_{2}\right)\right]+ \\
& \quad+\frac{\Gamma}{r^{2}} \frac{1}{m}\left[-i\left(\frac{d}{d r}+\frac{2}{r}\right)\left(\Psi_{2}-\psi_{2}\right)-2 i \frac{v}{r}\left(\Psi_{1}-\psi_{1}\right)+\frac{\Gamma}{r^{2}}\left(\Psi_{2}+\psi_{2}\right)\right]=2 m \psi_{2}, \\
& \left(E+\frac{\alpha}{r}\right)\left(\Psi_{2}+\psi_{2}\right)+\frac{d}{d r} \frac{1}{m}\left[\left(\frac{d}{d r}+\frac{2}{r}\right)\left(\Psi_{2}-\psi_{2}\right)+2 \frac{v}{r}\left(\Psi_{1}-\psi_{1}\right)+\frac{i \Gamma}{r^{2}}\left(\Psi_{2}+\psi_{2}\right)\right]=-2 m \psi_{2} .
\end{aligned}
$$

To get the non-relativistic equations for the big components $\Psi_{1}$ and $\Psi_{2}$, we sum the equations within each pair and neglect the small components. In this way we obtain

$$
\left\{\begin{array}{l}
\left(\frac{d^{2}}{d r^{2}}+\frac{2}{r} \frac{d}{d r}+\frac{\beta-\lambda r}{r}-\frac{2 v^{2}}{r^{2}}\right) \Psi_{1}-v \frac{2 r+\Gamma}{r^{3}} \Psi_{2}=0, \\
\left(\frac{d^{2}}{d r^{2}}+\frac{2}{r} \frac{d}{d r}+\frac{\beta-\lambda r}{r}-\frac{2 v^{2}}{r^{2}}-\frac{2}{r^{2}}-\frac{4 \Gamma}{r^{3}}-\frac{\Gamma^{2}}{r^{4}}\right) \Psi_{2}-2 v \frac{2 r+\Gamma}{r^{3}} \Psi_{1}=0,
\end{array}\right.
$$

where we have performed the change $i \Gamma \rightsquigarrow \Gamma$, and the following notations have been used:

$$
2 m E=-\lambda, \quad \lambda>0, \quad 2 m \alpha=\beta, \quad 2 v^{2}=j(j+1) \equiv L .
$$


This leads to the 4-th order equation for the function $\Psi_{1}(r)$ :

$$
\frac{d^{4}}{d r^{4}} \Psi_{1}+Q_{3} \frac{d^{3}}{d r^{3}} \Psi_{1}+Q_{2} \frac{d^{2}}{d r^{2}} \Psi_{1}+Q_{1} \frac{d}{d r} \Psi_{1}+Q_{0} \Psi_{1}=0
$$

where

$$
\begin{aligned}
Q_{3}= & -\frac{4}{2 r+\Gamma}+\frac{10}{r} \\
Q_{2}= & -2 \lambda+\frac{2 \Gamma \beta-24}{\Gamma} \frac{1}{r}+\frac{22-2 L}{r^{2}}-\frac{4 \Gamma}{r^{3}}-\frac{\Gamma^{2}}{r^{4}}+\frac{48}{\Gamma(2 r+\Gamma)}+\frac{8}{(2 r+\Gamma)^{2}} \\
Q_{1}= & \frac{-8 L+64-10 \Gamma^{2} \lambda-4 \Gamma \beta}{\Gamma^{2} r}+\frac{4 L-24+8 \Gamma \beta}{\Gamma r^{2}}+\frac{8-6 L}{r^{3}}-\frac{8 \Gamma}{r^{4}}-\frac{2 \Gamma^{2}}{r^{5}}+\frac{4 \Gamma^{2} \lambda+16 L-128+8 \Gamma \beta}{\Gamma^{2}(2 r+\Gamma)}-\frac{32}{\Gamma(2 r+\Gamma)^{2}} \\
Q_{0}= & \lambda^{2}+\frac{16 \Gamma^{2} \lambda+64 L+32 \Gamma \beta-2 \beta \lambda \Gamma^{3}}{\Gamma^{3} r}+\frac{-10 \Gamma^{2} \lambda-24 L-12 \Gamma \beta+\beta^{2} \Gamma^{2}+2 \lambda L \Gamma^{2}}{\Gamma^{2} r^{2}}+\frac{4 \Gamma^{2} \lambda+4 \Gamma \beta+8 L-2 \Gamma \beta L}{\Gamma r^{3}} \\
& +\frac{-4 \Gamma \beta+L^{2}+\Gamma^{2} \lambda-4 L}{r^{4}}-\frac{\Gamma^{2} \beta}{r^{5}}+\frac{-32 \Gamma^{2} \lambda-128 L-64 \Gamma \beta}{\Gamma^{3}(2 r+\Gamma)}+\frac{-32 L-8 \Gamma^{2} \lambda-16 \Gamma \beta}{\Gamma^{2}(2 r+\Gamma)^{2}} .
\end{aligned}
$$

The study of the solutions of this equation in the neighborhood of the regular singular point $r=-\Gamma / 2$ was studied in [13].

\section{The related KCC-geometrical approach}

Now we consider the problem of spin 1 particle with anomalous magnetic moment in the external Coulomb field by applying the Kosambi-Cartan-Chern geometrical theory. This geometrical study of the relevant system of differential equations is based on the use of KCC-invariants $[1,2,4,5]$.

In this approach, one considers a system of second order differential equations

$$
\dot{y}^{i}(r)+2 Q^{i}(r, x, y)=0,
$$

which corresponds to the the Euler-Lagrange equations for some differential system associated to a Lagrangian function $L$. In (15), the symbol $x^{i}$ designates so called coordinates, their derivatives in the argument $r$ are $y^{i}=d x^{i} / d r=\dot{x}^{i}$, and the quantities $Q_{i}$ are determined through some Lagrangian $L$, as follows.

$$
Q^{i}=\frac{1}{4} g^{i l}\left(\frac{\partial^{2} L}{\partial x^{k} \partial y^{l}} y^{k}-\frac{\partial L}{\partial x^{l}}+\frac{\partial^{2} L}{\partial y^{l} \partial r}\right), \quad g_{i j}=\frac{1}{2} \frac{\partial^{2} L}{\partial y^{i} \partial y^{j}} .
$$

The first and the second invariants, $\varepsilon^{i}(r, x, y)$ and $P_{j}^{i}$, defined by

$$
\begin{aligned}
& \varepsilon^{i}=\frac{\partial Q^{i}}{\partial y^{j}} y^{j}-2 Q^{i}, \\
& P_{j}^{i}=2 \frac{\partial Q^{i}}{\partial x^{j}}+2 Q^{s} \frac{\partial^{2} Q^{i}}{\partial y^{j} \partial y^{s}}-\frac{\partial^{2} Q^{i}}{\partial y^{j} \partial x^{s}} y^{s}-\frac{\partial Q^{i}}{\partial y^{s}} \frac{\partial Q^{s}}{\partial y^{j}}-\frac{\partial^{2} Q^{i}}{\partial y^{j} \partial r} .
\end{aligned}
$$

The second invariant $P_{j}^{i}$ describes the Jacobi stability of the system. There is an analogy between the equations of Riemannian geodesic deviation, and the ones governed by the second KCC-invariant:

$$
\frac{D^{2} \xi^{i}}{D s^{2}}=R_{k j l}^{i} \frac{d x^{k}}{d s} \frac{d x^{l}}{d s} \xi^{j}=-K_{j}^{i} \xi^{j} \sim \frac{D^{2} \xi^{i}}{D r^{2}}=P_{j}^{i} \xi^{j} .
$$

It is known that a pencil of geodesic curves which emerge from the same point $r_{0}$ converges (or diverges) if the real parts of all eigenvalues of the invariant $P_{j}^{i}$ are negative (or positive) ones.

We start with the radial system of two second-order differential equations

$$
\left\{\begin{array}{l}
\left(\frac{d^{2}}{d r^{2}}+\frac{2}{r} \frac{d}{d r}+2 m E+\frac{2 m \alpha}{r}-\frac{2 v^{2}}{r^{2}}-\frac{2 \Gamma}{r^{3}}-\frac{\Gamma^{2}}{r^{4}}\right) \Psi_{1}-v\left(\frac{2}{r^{2}}+\frac{\Gamma}{r^{3}}\right) \Psi_{2}=0, \\
\left(\frac{d^{2}}{d r^{2}}+\frac{2}{r} \frac{d}{d r}+2 m E+\frac{2 m \alpha}{r}-\frac{2 v^{2}+2}{r^{2}}\right) \Psi_{2}-2 v\left(\frac{2}{r^{2}}+\frac{\Gamma}{r^{3}}\right) \Psi_{1}=0 .
\end{array}\right.
$$

for two radial functions for the non-relativistic case. It should be emphasized that we shall follow the case of bound states, hence assuming $v=\sqrt{j(j+1) / 2}, \quad j=1,2,3, \ldots$. 
We further apply the notations $x^{i}=\Psi_{i}(r), y^{i}=(d / d r) \Psi_{i}(r)=\dot{\Psi}_{i}(r)$. Then, by comparing equations (??) and (15), one finds the relevant quantities $Q^{i}$ :

$$
\begin{aligned}
& Q^{1}\left(r, \Psi_{i}, \dot{\Psi}_{i}\right)=\frac{1}{2}\left(\frac{2}{r} \dot{\Psi}_{1}+\left(2 m \frac{\alpha+E r}{r}-\frac{2 v^{2}}{r^{2}}\right) \Psi_{1}-v \frac{2 r+\Gamma}{r^{3}} \Psi_{2}\right), \\
& Q^{2}\left(r, \Psi_{i}, \dot{\Psi}_{i}\right)=\frac{1}{2}\left(\frac{2}{r} \dot{\Psi}_{2}+\left(2 m \frac{\alpha+E r}{r}-\frac{2 v^{2}}{r^{2}}-\frac{2}{r^{2}}-\frac{4 \Gamma}{r^{3}}-\frac{\Gamma^{2}}{r^{4}}\right) \Psi_{2}-2 v \frac{2 r+\Gamma}{r^{3}} \Psi_{1}\right) .
\end{aligned}
$$

By direct calculation, according to the formulas (17), for the first invariant $\varepsilon^{i}$ we find two invariants:

$$
\begin{aligned}
& \left\{\begin{array}{l}
\varepsilon^{1}=\frac{v \Psi_{2}(\Gamma+2 r)}{r^{3}}+\Psi_{1}\left(-2 m E+\frac{2 v^{2}}{r^{2}}-\frac{2 m \alpha}{r}\right)-\frac{\dot{\Psi}_{1}}{r}, \\
\varepsilon^{2}=\frac{2 v \Psi_{1}(\Gamma+2 r)}{r^{3}}+\Psi_{2}\left(-2 m E+\frac{\Gamma^{2}}{r^{4}}+\frac{4 \Gamma}{r^{3}}+\frac{2\left(v^{2}+1\right)}{r^{2}}-\frac{2 m \alpha}{r}\right)-\frac{\dot{\Psi}_{2}}{r} ;
\end{array}\right. \\
& P_{j}^{i}=\left(\begin{array}{cc}
2 m \frac{\alpha+E r}{r}-\frac{2 v^{2}}{r^{2}} & -\frac{(2 r+\Gamma) v}{r^{3}} \\
-\frac{2(2 r+\Gamma) v}{r^{3}} & -\frac{\Gamma^{2}}{r^{4}}-\frac{4 \Gamma}{r^{3}}+2 m \frac{\alpha+E r}{r}-\frac{2\left(v^{2}+1\right)}{r^{2}}
\end{array}\right)
\end{aligned}
$$

The eigenvalues $\Lambda_{1}, \Lambda_{2}$ of the second invariant are given by the formula

$$
\Lambda_{1,2}=2 m E+\frac{1-2 v^{2}}{r^{2}}-\left(\frac{(\Gamma+2 r)^{2}}{2 r^{4}} \pm \frac{\sqrt{\left(\Gamma^{2}+2 r^{2}+4 \Gamma r\right)^{2}+8 v^{2} r^{2}(\Gamma+2 r)^{2}}}{2 r^{4}}\right)+\frac{2 m \alpha}{r}
$$

and the typical behavior of eigenvalues at different $j$ is presented in Figure 1.

(a)

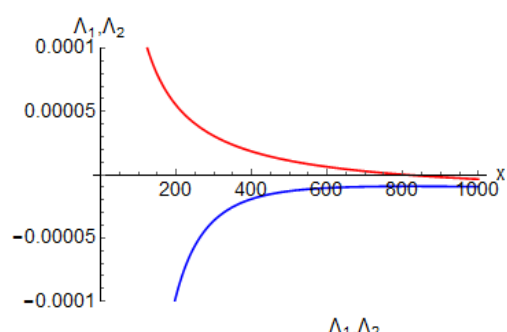

(b)
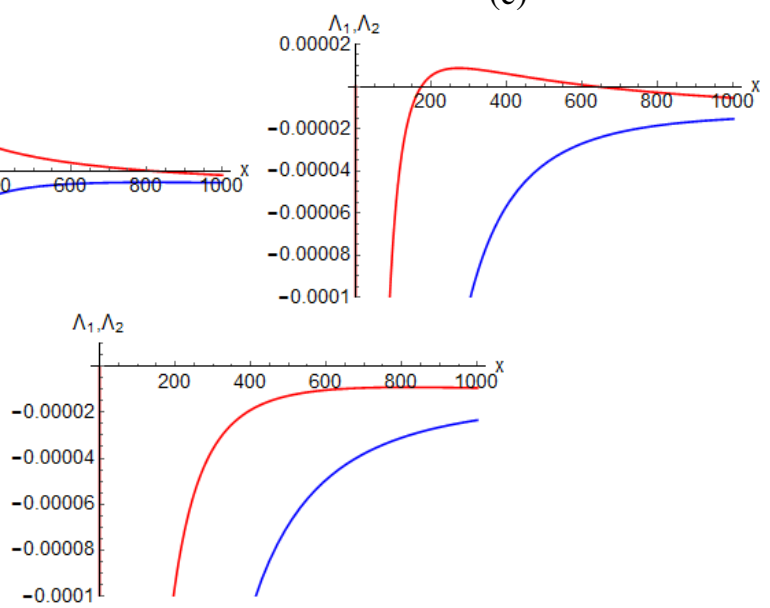

Figure 1. The dependencies of eigenvalues $\Lambda_{1}$ (red) and $\Lambda_{1}$ (blue) on radial coordinate $(x=m r)$ at different $j$ : (a) $j=1$, (b) $j=2$, (c) $j=3$. We used following parameters: $\Gamma m=1, E / m=-0.000009$.

We further specify their behavior near the singular points $r=0, r=\infty$, and $r=-\Gamma / 2$ :

$$
\begin{cases}r \rightarrow 0, & \rightsquigarrow \quad \Lambda_{1} \rightarrow \frac{2 m \alpha}{r}>0, \Lambda_{2} \rightarrow-\frac{\Gamma^{2}}{r^{4}}<0 ; \\ r \rightarrow \infty, & \rightsquigarrow \quad \Lambda_{1}, \Lambda_{2} \rightarrow 2 m E<0 ; \\ r \rightarrow-\frac{\Gamma}{2}, \quad \rightsquigarrow \quad \Lambda_{1} \rightarrow 2 m E-\frac{8 v^{2}}{\Gamma^{2}}-\frac{4 m \alpha}{\Gamma}<0, \quad \Lambda_{2} \rightarrow 2 m E-\frac{8\left(v^{2}-1\right)}{\Gamma^{2}}-\frac{4 m \alpha}{\Gamma}<0 .\end{cases}
$$

The behavior of the real parts of eigenvalues near the singular points $r=0, \infty,-\Gamma / 2$ correlates with the properties of solutions near the points $r=0, \infty,-\Gamma / 2$ for quantum mechanical bound states. 


\section{Additional KCC considerations}

For the states with parity $P=(-1)^{j}$, the differential system (8) writes:

$$
\begin{array}{ll}
-\left(\frac{d}{d r}+\frac{2}{r}\right) E_{2}-2 \frac{v}{r} E_{1}-\frac{\Gamma}{r^{2}} f_{2}=m f_{0}, & i\left(\epsilon+\frac{\alpha}{r}\right) E_{1}+i\left(\frac{d}{d r}+\frac{1}{r}\right) H_{1}=m f_{1}, \\
+i\left(\epsilon+\frac{\alpha}{r}\right) E_{2}-2 i \frac{v}{r} H_{1}-\frac{\Gamma}{r^{2}} f_{0}=m f_{2}, & -i\left(\epsilon+\frac{\alpha}{r}\right) f_{2}-\frac{d}{d r} f_{0}=m E_{2}, \\
-i\left(\epsilon+\frac{\alpha}{r}\right) f_{1}+\frac{v}{r} f_{0}=m E_{1}, & i\left(\frac{d}{d r}+\frac{1}{r}\right) f_{1}+i \frac{v}{r} f_{2}=-m H_{1},
\end{array}
$$

and by using the two non-differential equations, one can exclude two unknowns. As a direct consequence, by renoting the unknown remaining mappings, the complex SODE system reaches the more symmetrical form

$$
\left\{\begin{array} { c } 
{ \frac { d } { d r } g _ { 1 } = a g _ { 3 } + c g _ { 4 } } \\
{ \frac { d } { d r } g _ { 2 } = d g _ { 3 } + b g _ { 4 } }
\end{array} \quad \left\{\begin{array}{l}
\frac{d}{d r} g_{3}=A g_{1}+C g_{2} \\
\frac{d}{d r} g_{4}=D g_{1}+B g_{2},
\end{array}\right.\right.
$$

where the following rational functions are used:

$$
\begin{array}{ll}
a(r)=2 i v \frac{\epsilon r+\alpha}{r}, & c(r)=-\left(2 v^{2}+r^{2}\right), \\
d(r)=i \frac{(\epsilon r+\alpha)^{2}-r^{2}}{r^{2}}, & b(r)=-\frac{v(\epsilon r+\alpha)}{r}, \\
A(r)=-i \frac{v(\epsilon r+\alpha)}{r^{3}}, & C(r)=i \frac{\left(2 v^{2}+r^{2}\right)}{r^{2}}, \\
D(r)=\frac{(\epsilon r+\alpha)^{2}-r^{2}}{r^{4}}, & B(r)=-\frac{2 v(\epsilon r+\alpha)}{r^{3}} .
\end{array}
$$

We shall further consider the 2-nd order extension of the real sibling of the system (21).

We further denote the independent variable as $r \rightsquigarrow t$ (and $\frac{d}{d r} \leadsto \frac{d}{d t}$ ), and the unknown complex mapping $g: \mathbb{R} \rightarrow \mathbb{C}^{4}$ as

$$
g=\left(g_{1}, g_{2}, g_{3}, g_{4}\right)^{T} \rightsquigarrow x=\left(x_{1}, x_{2}, x_{3}, x_{4}\right)^{T} .
$$

Then the considered differential system has the brief form

$$
\dot{x}=M x
$$

where $M=\left(\begin{array}{ll}0 & P \\ Q & 0\end{array}\right)$ has the off-diagonal blocks $P=\left(\begin{array}{cc}a & c \\ b & d\end{array}\right)$ and $Q=\left(\begin{array}{ll}A & C \\ B & D\end{array}\right)$, with the components

$$
\begin{array}{llll}
a=2 i \beta_{1}, & d=i \beta_{3}, & A=-\frac{i}{t^{2}} \beta_{1}, & C=\frac{i}{t^{2}} \beta_{2} \\
c=-\beta_{2}, & b=-\beta_{1}, & D=\frac{\beta_{3}}{t^{2}}, & B=-\frac{2}{t^{2}} \beta_{1},
\end{array}
$$

$\beta_{1}=\frac{v(\varepsilon t+\alpha)}{t}, \beta_{2}=2 v^{2}+t^{2}$, and $\beta_{3}=\frac{(\varepsilon t+\alpha)^{2}-t^{2}}{t^{2}}$. We note that $a, d, A, C$ are purely-imaginary mappings, while $c, b, D, B$ are real.

We note that, via $\beta_{1}, \beta_{2}, \beta_{3}$, the complex scalar functions $a, b, c, d, A, B, C, D$ essentially depend on the following parameters:

1. $\epsilon \in(0,1) \cup(1, \infty)$ - the domain corresponding to two physically substantially different regions, of which most interesting is the first (bound states), e.g., $\epsilon$ inferior close to 1 .

2. $v=\sqrt{j(j+1) / 2}, j=1,2,3, \ldots$.

3. $\alpha=\frac{1}{137}$.

Generally, $t \in(0, \infty)$; in applications one chooses $t \in(0, T), T \in \overline{1,10}$ - where the interesting values are the ones $t \in\left(0, \frac{T}{2}\right)$.

As well, a meaningless particular choice is, e.g., in the vicinity of $j=1,(v=1), \epsilon=1-10^{-7}$. 


\subsection{The 8D real fist-order differential system}

The KCC (Kosambi-Cartan-Chern) framework applies to our case, after re-writing the system in $\mathbb{R}^{8} \equiv$ $\mathbb{C}^{4}$. We denote $x_{k}=x^{\prime}{ }_{k}+i x^{\prime \prime}{ }_{k}(k \in \overline{1,4})$, with $x^{\prime}{ }_{k}, x^{\prime \prime}{ }_{k}$ real functions.

As well, the matrix $M$ changes, and for

$$
a=i a^{\prime}, \quad d=i d^{\prime}, \quad A=i A^{\prime}, \quad C=i C^{\prime},
$$

the two $4 \times 4$ off-diagonal blocks of the obtained $8 \times 8$ real matrix are

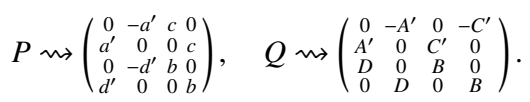

Indeed, the complex system $\left(\begin{array}{c}\dot{x}_{1} \\ \dot{x}_{2} \\ \dot{x}_{3} \\ \dot{x}_{4}\end{array}\right)=\left(\begin{array}{cccc}0 & 0 & i a^{\prime} & c \\ 0 & 0 & 1 d^{\prime} & b \\ i A^{\prime} & i C^{\prime} & 0 & 0 \\ D & B & 0 & 0\end{array}\right)\left(\begin{array}{l}x_{1} \\ x_{2} \\ x_{3} \\ x_{4}\end{array}\right)$ is equivalent to the two $4 \mathrm{D}$ real subsystems

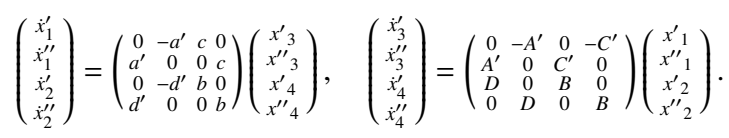

For convenience, we further denote the real vector of unknown scalar functions as:

$$
\left(x^{\prime}{ }_{1}, x^{\prime \prime}{ }_{1}, \ldots, x^{\prime}{ }_{4}, x^{\prime \prime}{ }_{4}\right)^{T} \rightsquigarrow x=\left(x_{1}, \ldots, x_{8}\right)^{T} .
$$

We shall further study two straightforward extensions of the 1-st order real differential system, and identify necessary conditions for flattening its parametrized solutions, namely the existence of autonomous diffeomorphisms $(t, x) \rightarrow(t, \tilde{x})$ which transforms them to parametrized lines. This is relevant in identifying the cases when the initial system does not allow such a local path flattening.

\subsection{The non-autonomous KCC framework}

In KCC theory - (intensively studied by Antonelli, Belvilacqua, Rutz, and Bucataru [4], Sabău, Neagu a.o.) - one emerges from a real differential system of the form

$$
\ddot{x}+g(t, x, y)=0, \quad y=\dot{x} \in T_{x}\left(\mathbb{R}^{n}\right) .
$$

The straightening of the solutions of the system under time-preserving diffeomorphisms is ensured by the simultaneous vanishing of the following five KCC invariants, and used in the study of structural (Jacobi) stability:

- The first KCC invariant is

$$
\varepsilon^{i}(x, y)=\frac{1}{2} g_{s}^{i} y^{s}-g^{i}, \quad \text { where } g_{s}^{i}=\frac{\partial g^{i}}{\partial y^{s}} .
$$

- The second KCC invariant detects the gathering/dissipating of the concurrent sheaves of solutions:

$$
P_{j}^{i}=-\frac{\partial g^{i}}{\partial x^{j}}-\frac{1}{2} g^{s} g_{j s}^{i}+\frac{1}{2} \frac{\partial g_{j}^{i}}{\partial x^{s}} y^{s}+\frac{1}{4} g_{s}^{i} g_{j}^{s}+\frac{1}{2} \frac{\partial g_{j}^{i}}{\partial t},
$$

where $g_{j s}^{i}=\frac{\partial g_{j}^{i}}{\partial y^{s}}$.

- the third KCC invariant

$$
R_{j k}^{i}=\frac{1}{3}\left(\frac{\partial P_{j}^{i}}{\partial y^{k}}-\frac{\partial P_{k}^{i}}{\partial y^{j}}\right)
$$

In the Finsler case, for the equations of geodesics, $R_{j k}^{i}$ is the the torsion of the Berwald connection (the strong curvature of the nonlinear connection). 
- the fourth KCC invariant

$$
B_{j k l}^{i}=\frac{\delta R_{j k}^{i}}{\delta y^{l}} .
$$

In the Finsler case, this is the Riemann-Christoffel curvature tensor of the Berwald connection.

- the fifth KCC invariant is

$$
D_{j k l}^{i}=\frac{\partial^{3} g^{i}}{\partial y^{j} \partial y^{k} \partial y^{l}} .
$$

In the Finsler case, this is the Douglas tensor, the last curvature of the Berwald connection.

\subsubsection{The Geometric Dynamics extension of the first-order SODE}

The form of our 2-nd order differential extension is:

$$
\ddot{x}=\varphi(t) x+\psi(t) y, \quad y=\dot{x},
$$

with skew-symmetric (curl-type, gyroscopic) $\psi(t)$ and evolutionary (modified gradient) type $\varphi(t)$. Specifically, since $\dot{x}=M x$ and $\ddot{x}=\dot{M} x+M \dot{x}$, we yield the Geometric Dynamics 2-nd order extension

$$
\ddot{x}=\left(\dot{M}+M^{T} M\right) x+\left(M-M^{T}\right) y, \quad \text { where } y=\dot{x} .
$$

This can be written as

$$
\ddot{x}+g(x, y, t)=0,
$$

where

$$
g(x, y, t)=-\Delta y-\sigma x, \quad \Delta=M-M^{T}, \quad \sigma=\dot{M}+M^{T} M .
$$

We note that $\Delta$ and $\sigma$ are real matrices depending on time only.

For computing the KCC-invariants, in the case of the GD extension of dimension 8, the situation simplifies, due to the specific form of $g^{i}(t, x, y)$, briefly written as $g=-\Delta y-\sigma x$, where

$$
\Delta=M-M^{T}, \quad \sigma=\dot{M}+M^{T} M,
$$

are time only - dependent matrices. This leads to

$$
g_{r}^{i}=-\Delta, \quad \frac{\partial g^{i}}{\partial x^{j}}=-\sigma, \quad \frac{\partial g_{j}^{i}}{\partial x^{r}}=0, \quad g_{j r}^{i}=0, \quad \frac{\partial g_{j}^{i}}{\partial t}=-\dot{\Delta} .
$$

Concluding, we infer the following results.

Theorem 4.1. The five geometric invariants of the 2-nd order GD extension of the 1-st order magnetic spin 1 particle system, are:

$$
\varepsilon=\sigma x+\frac{1}{2} \Delta y, \quad P=\sigma-\frac{1}{4} \Delta^{2}-\frac{1}{2} \cdot \Delta, \quad R=0, B=0, D=0 .
$$

Corollary 4.1. A necessary condition for the existence of a time-independent diffeomorphism which straightens the solutions of the system exists, is $\sigma=\delta=0$.

This comes to $M$ being symmetric and subject to the SODE $\dot{M}=-M^{T} M$.

\subsubsection{The natural extension of the first-order SODE}

Since the system $\dot{x}=M x$ has the particular matrix of the form $M=\left(\begin{array}{ll}0 & P \\ Q & 0\end{array}\right)$, with $x \in C^{2}\left(I, \mathbb{C}^{4}\right)$, by denoting $z^{\prime}=\left(x_{1}, x_{2}\right)^{T}$ and $z^{\prime \prime}=\left(x_{3}, x_{4}\right)^{T}$, the system becomes

$$
\dot{z}^{\prime}=P z^{\prime \prime}, \quad \dot{z}^{\prime \prime}=Q z^{\prime} .
$$

Then, by derivation, one gets the following form of the subsystems of the natural extension of the initial 1-st order system, due to its particular form: 
a) For $P$ invertible,

$$
\ddot{z}^{\prime}=\dot{P} P^{-1} \dot{z}^{\prime}+P Q z^{\prime}
$$

b) For $Q$ invertible,

$$
\ddot{z}^{\prime \prime}=\dot{Q} Q^{-1} \dot{z}^{\prime \prime}+Q P z^{\prime \prime} .
$$

The systems a) and b) are symbolically equivalent by means of the interchange $P \leftrightarrow Q$, and they form together the normal 2-nd order (complex) extension of the initial SODE, leading to the real one like in case I. We note that both the subsystems have the general form

$$
\ddot{x}+g(t, x, \dot{x})=0, \quad g=R \dot{x}+S x,
$$

with $t$-dependent mappings $R, S$. Taking into account that

$$
\begin{gathered}
{\left[\frac{\partial g^{i}}{\partial y^{r}}\right]=\left[g_{r}^{i}\right]=R,\left[\frac{\partial g^{i}}{\partial x^{j}}\right]=S,\left[\frac{\partial^{2} g^{i}}{\partial y^{r} \partial y^{j}}\right]=\left[\frac{\partial^{2} g^{i}}{\partial x^{r} \partial y^{j}}\right]=0,} \\
{\left[g_{r}^{i} r_{j}^{r}\right]=R^{2},\left[\frac{\partial g_{j}^{i}}{\partial t}\right]=\frac{\partial R}{\partial t}=\dot{R} .}
\end{gathered}
$$

we infer the KCC invariants, as follows

Theorem 4.2. The five KCC invariants of the SODE (22) are:

$$
\varepsilon=-\frac{1}{2} R \dot{x}-S X, \quad\left[P_{j}^{i}\right]=-S+\frac{1}{4} R^{2}=\frac{1}{2} \dot{R}, \quad\left[R_{j}^{i} k\right]=\left[B_{j k l}^{i}\right]=\left[D_{j}^{i} k l\right]=0 .
$$

Hence the KCC straightening necessary condition becomes $R=S=0$.

E.g., the 2-nd order subsystem for $z^{\prime}$ has $R=\dot{P} P^{-1}$ and $S=P Q$. The condition requires that $P$ should be constant non-singular, and $Q$ vanishing, which trivializes the 1 -st order initial system, $\dot{z}^{\prime}=P z^{\prime \prime}$, with $\dot{z}^{\prime \prime}=0$. Hence with $\dot{z}^{\prime}=$ const and $z^{\prime \prime}$ null, the solutions are exactly affine $t$-parametrized lines.

\subsection{Related KCC-stability issues}

We note that the considered 1-st order SODE is a linear homogeneous and non-autonomous dynamical system. This implies the vanishing of the last three KCC-invariants.

The vanishing of the first invariant would imply the trivialization of the system, which will allow only straight lines. But the matrix $M(t)$ which governs the system $\dot{x}=M x$ cannot be constant, due to the form of the coefficients and to the physically meaningful domains of the parameters $\alpha, \epsilon, v$, which lead to non-trivial $\beta_{1}, \beta_{2}, \beta_{3}$ and further everywhere nonzero $P$ and $Q$. Hence, due to the nontrivial 1-st invariant, none of the two considered 2-nd order extensions allow trivializing diffeomeorphisms: (i) in the first case, due to the non-attendable symmetry condition (e.g., $a=A$ leads to a contradiction), and (ii) in the second case, since none of the meaningful instances of the matrices $P, Q$ may ever vanish.

Accordingly, the solutions of the 2-nd order extensions (which contain the affinely parametrized solutions of the initial SODE), according to the KCC theory, can never be geodesics of structures of Finsler, Riemann or Euclidean spaces. This shows a peculiar complex facet of the studied SODE.

As for the second invariant, the spectral properties of the tensor $\left[P_{j}^{i}\right]$ indicate the converging/diverging sheaf properties of the behavior of the solutions: since the KCC stability is a weaker condition than the stability of periodic orbits given by the sign of the Floquet exponents (characteristic multipliers), and is provided by the spectral properties of the second invariant $\left[P_{j}^{i}\right]$, due to the equality $\frac{D^{2} \xi^{i}}{d t^{2}}=P_{r}^{i} \xi^{r}$, where $D$ is the KCC-covariant differential.

A further concern has in view the spectral properties of this operator for the two extensions, and its relevance on the subclass of solutions of the primary 1-st order SODE.

Acknowledgements. The present work was developed under the auspices of the Project F19M-032 of Belarusian Republican Foundation for Fundamental Research for young scientists and of the Project BRFFR No. A18-RA-015/2019-2020, within the cooperation framework between Romanian Academy and Belarusian Republican Foundation for Fundamental Research. 


\section{References}

[1] P.L. Antonelli, Equivalence problem for systems of second order ordinary differential equations (In: M. Hazewinkel (Ed.) Encyclopedia of Mathematics, Dordrecht: Kluwer Academic Publishers, 2000) 546 pp.

[2] P.L. Antonelli, I. Bucataru, KCC theory of a system of second order differential equations (In: P.L. Antonelli (Ed.) Handbook of Finsler Geometry, Springer, 2003) 1-66.

[3] P.L. Antonelli, L. Bevilacqua, S.F. Rutz, Theories and models in symbiogenesis, Nonlinear Analysis, Real World Applications 4, 743-753 (2003).

[4] P.L. Antonelli, I. Bucataru, New results about the geometric invariants in KCC-theory, An.St. Univ. "Al.I.Cuza" Iasi. Mat. N.S. 47, 405-420 (2001).

[5] Gh. Atanasiu, V. Balan, N. Brinzei, and M. Rahula, Differential Geometry of The Second Order and Applications: Miron-Atanasiu Theory (in Russian) (Librokom Eds., Moscow, 2010) $256 \mathrm{pp}$.

[6] V. Balan, Jacobi structural stability for a SODE biochemical model, Proc. of The XIth Int.Conf. "Man in The Knowledge Based Organization", 23-25 Nov. (2006), Sibiu, "Nicolae Balcescu" Land ForcesAcademy Editors, 472-478 (2006).

[7] V. Balan, M. Neagu, Jet geometrical extension of the KCC-invariants, Balkan J. Geom. Appl. 15(1), 8-16 (2010); arXiv:0906.2903v1 [math.DG] 16 Jun2009.

[8] H.C. Corben and J. Schwinger, The electromagnetic properties of mesotrons, Physical Review 58, 953 (1940).

[9] V.V. Kisel, E.M. Ovsiuyk and V.M. Red'kov, On the wave functions and energy spectrum for a spin 1 particle in external Coulomb field, Nonlinear Phenomena in Complex Systems 13(4), 352-367 (2010).

[10] V.V. Kisel, E.M. Ovsiyuk, Ya.A. Voynova and V.M. Red'kov, Quantum mechanics of spin 1 particle with quadrupole moment in external uniform magetic field, Problems of Physics, Mathematics and Thechnics 32(3), 18-27 (2017).

[11] V.V. Kisel, V.M. Red'kov, E.M. Ovsiyuk, Wave functions and energy spectrum for spin 1 particle in Coulomb field, Doklady Natsionalnoi Akademii Nauk Belarusi 55(1), 50-55 (2011).

[12] V.V. Kisel, Ya.A. Voynova, E.M. Ovsiyuk, V. Balan and V.M. Red'kov, Spin 1 particle with anomalous magnetic moment in the external uniform magnetic field, Nonlinear Phenomena in Complex Systems 20(1), 21-39 (2017).

[13] A. Koralkov, Ya. Voynova, N. Krylova, E. Ovsiyuk, V. Balan, Vector particle with electric quadrupole moment inexternal Coulomb field, BSG Proceedings 27, 80-106 (2020).

[14] E.M. Ovsiyuk, Quantum Kepler problem for spin 1/2 particle in spaces on constant curvature. I. Pauli Theory, E.M. Ovsiyuk, Nonlinear Phenomena in Complex Systems 14(1), 14-26 (2011).

[15] E.M. Ovsiyuk, V.V. Kisel, V.M. Red'kov, Maxwell Electrodynamics and Boson Fields in Spaces of Constant Curvature (Nova Sci. Publishers, New York, 2014) 498 pp.

[16] E.M. Ovsiyuk, V.V. Kisel, O.V. Veko, Ya.A. Voynova, V. Balan and V.M. Red'kov, Elementary particles with internal structure in external field. II. Physical Problems (Nova Sci. Publishers, USA, 2018) 402 pp.

[17] E.M. Ovsiyuk, V.V. Kisel, O.V. Veko, Ya.A. Voynova, V. Balan and V.M. Red'kov, Elementary particles with internal structure in external field. I. General Theory (Nova Sci. Publishers, USA, 2018) 404 pp.

[18] E.M. Ovsiyuk, O.V. Veko, Ya.A. Voynova, A.D. Koral'kov, V.V. Kisel, V.M. Red'kov, On describing bound states for a spin 1 particle in the external Coulomb field, Balkan Society of Geometers Proceedings 25, 59-78 (2018). 
[19] E.M. Ovsiyuk, Ya.A. Voynova, V.V. Kisel, V. Balan, V.M. Red'kov, Spin 1 particle with anomalous magnetic moment in the external uniform electric field (In S. Griffin (Eds.) Quaternions: Theory and Applications, Nova Science Publishers, USA, 2017) 47-84.

[20] E.M. Ovsiyuk, Ya.A. Voynova, V.V. Kisel, V. Balan, V.M. Red'kov, Spin 1 particle with anomalous magnetic moment in the external uniform electric field, Nonlinear Phenomena in Complex Systems 21(1), 1-20 (2018).

[21] E.M. Ovsiyuk, Ya.A. Voynova, V.V. Kisel, V. Balan, V.M. Red'kov, Techniques of projective operators used to construct solutions for a spin 1 particle with anomalous magnetic moment in the external magnetic field (In S. Griffin (Eds.), Quaternions: Theory and Applications, Nova Sci. Publishers, USA, 2017) 11-46.

[22] V.A. Pletjukhov, V.M. Red'kov, V.I. Strazhev, Relativistic wave equations and intrinsic degrees of freedom (Belarusian Science, Minsk) $328 \mathrm{pp}$.

[23] V.M. Red'kov, Fields in Riemannian Space and the Lorentz Group (Belarusian Science, Minsk, 2009) 486 pp.

[24] V.M. Red'kov, Tetrad Formalism, Spherical Symmetry and Schrödinger basis (Belarusian Science, Minsk, 2011) 339 pp.

[25] A. Ronveaux, Heun's differential equation (Oxford University Press, 1995) 380 pp.

[26] V.S. Sabău, Systems biology and deviation curvature tensor (Lecture notes, University of Alberta, Canada, 2001).

[27] S.Yu. Slavyanov, W. Lay, Special functions. A unified theory based on singularities (Oxford University Press, 2000) 312 pp.

[28] A. Shamaly, A.Z. Capri, Unified theories for massive spin 1 fields, Canadian Journal of Physics 51(14), 1467-1470 (1973).

[29] I.E. Tamm, Motion of mesons in electromagnetic fields, Dokl. Akad. Nauk SSSR 29, 551 (1940).

[30] Constantin Udrişte, Massimiliano Ferrara, Dumitru Opriş, Economic Geometric Dynamics (Geometry Balkan Press, Bucharest, 2004) 270 pp. 